\title{
Sfide e limiti del veganismo. Vegefobia, mass media e mercato Nicola Righetti
}

In Bertuzzi, N., Reggio, M. (2019). «Smontare la gabbia. Anticapitalismo e movimento di liberazione animale», Mimesis, pp. 17-26.

Quando un movimento controculturale entra a contatto con la cultura mainstream circostante, gli attriti necessariamente causati dalla critica sferrata ai valori più diffusi, ai modi di pensare più consolidati, alle abitudini e ai rituali quotidiani, non possono che attivare, in men che non si dica, una serie di reazioni difensive. Il veganismo non fa eccezione. Questo non dovrebbe stupire, se solo si considera la radicalità della sua proposta: modificare valori e significati sedimentati nel tempo intorno alla vita animale. E poco importa il metodo seguito per raggiungere l'obiettivo. Attuare cambiamenti nei consumi personali per tentare di modificare equilibri culturali, sociali ed economici - secondo l'idea che una moltitudine di cambiamenti individuali possano, a valanga, sortire effetti strutturali - non basta a far passare inosservato il tentativo "sovversivo" e a ingannare il sistema.

La dialettica tra cambiamento e resistenza al cambiamento descrive nel modo più elementare i rapporti tra un movimento controculturale come il veganismo e il suo contesto sociale ${ }^{1}$. È allora di fondamentale importanza individuare quali strategie configurino tale resistenza. Anche perché il veganismo non può evitare di confrontarsi con esse, a meno di non abdicare ai propri ideali. Come si cercherà di illustrare, questi meccanismi difensivi si manifestano anzitutto come resistenza alla diffusione delle istanze politiche vegane. Ma non è tutto. A fronte di esplicite forme di contrasto si profilano meccanismi più sottili, meno evidenti, apparentemente liberali ma non per questo meno efficaci, che possono neutralizzare le istanze di cambiamento, addomesticandole. Se in una prima fase di contatto tra cultura vegana e mainstream si possono più facilmente individuare strategie del primo tipo, oggi assumono una maggiore rilevanza quelle del secondo tipo.

${ }^{1}$ Cfr. John Milton Yinger, «Contraculture and Subculture», in «American Sociological Review», vol. 25, n. 5, 1960, pp. 625-635. 
Un'analisi delle strategie di resistenza al veganismo sarebbe infine quasi inutile se non fosse accompagnata da un'analisi delle caratteristiche del veganismo stesso. Infatti, quest'ultimo potrebbe involontariamente offrire delle affordance alle strategie che vi si oppongono. Occorre chiedersi, in altre parole, se il veganismo non sia caratterizzato da elementi di debolezza su cui le strategie di resistenza possano fare leva, ostacolando il perseguimento degli obiettivi desiderati. Nel corso di questo contributo si articolerà una riflessione su questi temi.

\section{Il veganismo diventa mainstream}

È impervia e ricca di ostacoli la strada di chi mira al cambiamento. Tra le strategie che difendono lo status quo, la più semplice è la marginalizzazione delle istanze innovative e dei loro portavoce. Silenziate le idee che potrebbero interferire con l'organizzazione tessuta nel corso del tempo, l'ordine sociale può continuare a riprodursi.

La storia insegna una grande varietà di meccanismi messi a punto a questo scopo, accumunati tutti dalla medesima volontà di reprimere le voci "altre": dalla persecuzione e soppressione fisica a forme più raffinate, ma non per questo meno "violente", di censura e contenimento delle voci critiche. Oggi, tuttavia, nessun braccio armato minaccia l'esistenza dei vegani. Nessun potere di censura, nel senso proprio del termine, li mantiene segregati nel silenzio, ai margini della società, impedendo loro di prendere parola. Anzi, almeno per certi versi la situazione sembra essere persino opposta. Difatti, non è forse vero che il veganismo ha conosciuto negli ultimi anni una straordinaria popolarità? Tutti i giornali ne hanno parlato. Anzi, davanti al veganismo si è srotolato il tappeto rosso, promuovendolo negli ambienti più in vista dello star system internazionale. La stessa Vegan Society, poco avvezza alle luci della ribalta, ha constatato che i mass media non avevano mai dedicato tanta attenzione al veganismo come negli ultimi anni. In un brevissimo arco di tempo, il piccolo manipolo di difensori della vita animale era passato dall'essere poco più che un club esclusivo di cui nessuno si interessava, ad un fenomeno di massa presente sulle pagine di tutti i giornali ${ }^{2}$. Le statistiche degli ultimi anni hanno segnalato un incremento del numero dei vegani in

\footnotetext{
${ }^{2}$ Cfr. The Vegan Society, «Ripened by human determination. 70 years of The Vegan Society», 2014, https://www.vegansociety.com/sites/default/files/uploads/Ripened\%20by\%20human\%20determination.pdf, p. 1 .
} 
Italia, addirittura triplicati nel giro di un anno $^{3}$, fenomeno in cui tanti blogger hanno creduto di vedere l'alba del cambiamento sperato. Per non parlare, ovviamente, dell'arrivo dei prodotti vegani sugli scaffali dei supermercati.

Un cambiamento davvero straordinario si è quindi realizzato nel rapporto tra veganismo e società e nulla lascia intravedere forme di radicale repressione o censura. Al contrario, il contesto sembra segnalare una completa accettazione del veganismo, una vera e propria integrazione nel mainstream.

Missione compiuta, quindi? Non proprio. Occorre infatti ammettere che il cambiamento desiderato dai vegani non è, in realtà, mai arrivato. Nonostante il suo debutto in società, il veganismo si è andato semplicemente affiancando alla cultura e allo stile di vita tradizionali. Senza trasformarli radicalmente, anzi, senza scalfirli particolarmente.

L'immagine del cambiamento realmente avvenuto, piuttosto, è ben rappresentata dallo scaffale del supermercato: alle confezioni di latte e bistecche si sono affiancate, non certo sostituite, quelle dei latti vegetali e delle bistecche di soia, in una pacifica convivenza che non rinvia ad alcun conflitto né contraddizione apparenti. La democrazia dei consumi, d'altronde, ha spazio a sufficienza per tutti coloro che abbiano sufficiente potere d'acquisto, non conosce barriere ideologiche insuperabili che non possano essere ammortizzate dalla cornucopia dei suoi prodotti, la sua inclusività è massima e nessuna nicchia potenzialmente profittevole viene esclusa.

Paradossalmente, tuttavia, a fronte di questa accresciuta popolarità e offerta di alternative vegetali, le statistiche più recenti segnalano un crollo nel numero dei vegani, tanto vertiginoso e repentino quanto è stato il loro aumento ${ }^{4}$. Che l'ingresso del veganismo nella cultura e nel mercato mainstream si siano accompagnati ad un crollo nelle adesioni rappresenta, certo, soltanto un indizio che qualcosa sta accadendo; ma sommato al contesto appena tratteggiato spinge a una riflessione: cosa sta accadendo nel punto di giunzione tra veganismo e società?

Non si può, d'altra parte, fare a meno di chiedersi come sia possibile che un movimento portatore di istanze tanto radicali e culturalmente rivoluzionarie venga accolto così facilmente nel mainstream che esso critica e vuole trasformare. Non dovremmo trovare, al contrario, armi spianate ad attenderlo al varco? In effetti, basta

${ }^{3}$ Cfr. Eurispes, «Rapporto Italia 2017», 2017.

${ }^{4}$ Cfr. Eurispes, «Rapporto Italia 2018», 2018. 
sollevare il velo della superficiale popolarità e dell'apparente integrazione per portare alla luce gli attesi meccanismi di preservazione dello status quo.

\section{Vegefobia e rapporti con i media}

Definiamo "strategie contrastive" di resistenza al veganismo quelle più facilmente percepibili e identificabili. Si manifestano sia sui mass media, sia nella vita quotidiana dei vegani. Sono segnalate da un esplicito attrito tra veganismo e cultura mainstream. Sebbene non assumano mai le forme più estreme della censura radicale e della persecuzione fisica, innalzano una barriera protettiva a difesa delle tradizionali concezioni culturali di animale e di rapporto tra uomo e animale. Attacchi mirati a squalificare, stigmatizzare ed a marginalizzare le istanze etico-politiche vegane, caratterizzano queste strategie. I mattoni con cui innalzano i loro muri sono forgiati con materiale culturale solidificato nel corso del tempo, le fondamenta su cui poggiano affondano nell'antropocentrismo, nella cultura individualista, secolarizzata e consumista.

La superiore dignità attribuita all'uomo rappresenta il fondamento delle più tipiche manifestazioni di queste strategie, che squalificano istantaneamente le istanze vegane come espressioni di fanatismo ${ }^{5}$. Pur formalmente libero di esprimersi, davanti a un muro di pregiudizio il veganismo viene squalificato, delegittimato, svalutato, marginalizzato. Lo stesso accade a colui che ne propone le idee. Mentre la censura agisce preventivamente sulla fonte della comunicazione giudicata problematica, il pregiudizio agisce sul destinatario e colpisce la fonte solo indirettamente, mediante il feedback stigmatizzante inviato dal destinatario stesso, che è a sua volta impedito nella piena comprensione del messaggio a causa del proprio pregiudizio.

Questo processo comunicativo di carattere generale si manifesta a diversi livelli della vita quotidiana e della comunicazione mass mediatica, tra loro in un rapporto di reciproca interazione. Proprio dall'analisi dei media è inizialmente emersa la peculiare costellazione di pregiudizi contro il veganismo e i vegani definita "vegefobia" ${ }^{6}$ Alcuni

\footnotetext{
${ }^{5}$ Cfr. N. Righetti, «Tra sacro e vegano», Ferrari Editore, Corigliano - Rossano 2018.

${ }^{6}$ Cfr. Matthew Cole e Karen Morgan, «Vegaphobia: Derogatory Discourses of Veganism and the Reproduction of Speciesism in UK National Newspapers», in «British Journal of Sociology», n. 62, vol. 1, 2011, pp. 134-153 (trad. it. in http://it.vegephobia.info/index.php?post/2012/05/21/Vegafobia-di-M.Cole-eK.-Morgan).
} 
dei pregiudizi compresi in questa particolare costellazione sono stati riscontrati anche sulla stampa italiana ${ }^{7}$.

La vegefobia mediatica promuove un'immagine negativa dei vegani e del veganismo in molti modi. Per esempio, descrivendo i vegani come fanatici e seminando timori relativamente alla salubrità dell'alimentazione vegana. Il timore appare riguardare non tanto l'alimentazione vegana di per sé, ma quella seguita "troppo a lungo". Come a dire, preoccupa quella adottata per motivazioni etiche, non quella seguita dopo le scorpacciate natalizie e sospesa una volta tornati in forma.

Il veganismo non viene danneggiato soltanto dai pregiudizi esplicitamente espressi, ma anche dai contenuti sottaciuti. Si è scritto tanto di veganismo sui giornali, molto più raramente delle sue motivazioni. Questa miscela di pregiudizi e silenzi emerge dalla cultura antropocentrica e allo stesso tempo la protegge e la rafforza. Il mondo vegan ne esce stigmatizzato, con il possibile effetto di allontanare potenziali sostenitori.

Il concetto di vegefobia, da solo, non basta però a spiegare i meccanismi sottostanti la comunicazione sul veganismo. La cultura antropocentrica fornisce la materia prima per forgiare il discorso anti-vegano, colto tanto bene dal concetto di vegefobia, ma sono le strutture del sistema dei mass media a permettere la sua diffusione. La selezione delle notizie e testimonianze rilevanti, nonché il modo di raccontarle, avviene secondo le logiche del sistema mediatico, che un movimento dovrebbe prendere in considerazione ${ }^{8}$. Solo gli attori sociali capaci di accreditarsi vedono crescere le opportunità di far ascoltare la propria voce. Tuttavia, il veganismo, a causa della sua natura frammentata, individualistica e in definitiva priva di struttura organizzativa - caratteristiche comuni ai «lifestyle movement» ${ }^{9}$ - manca della capacità di interagire efficacemente con il sistema mediatico, così risultando in balia delle notizie scritte sul suo conto, privo di controllo, per quanto parziale, sulla sua stessa immagine proiettata dai media.

Ciò non manca di provocare conseguenze comunicative, culturali e politiche. L'origine del problema, tuttavia, è di tipo organizzativo. Infatti, lo stesso problema non

\footnotetext{
${ }^{7}$ Cfr. Nicola Righetti, «L'inchiostro digitale è vegano? La rappresentazione del veganismo sulla stampa», in «Cambio. Rivista Sulle Trasformazioni Sociali», n. 11, 2016, pp. 181-194.

${ }^{8}$ Cfr. William A. Gamson e Gadi Wolfsfeld, «Movements and Media as Interacting Systems», in «The Annals of the American Academy of Political and Social Science», n. 528, maggio 1993, pp. 114-125.

${ }^{9}$ Cfr. Ross Haenfler, Brett Johnson, Ellis Jones, «Lifestyle Movements: Exploring the Intersection of Lifestyle and Social Movements», in «Social Movement Studies», vol. 11, n. 1, 2012, pp. 1-20.
} 
riguarda in modo così rilevante le associazioni animaliste più organizzate, che si dimostrano in grado di interagire con la stampa più efficacemente, veicolando i propri valori e idee di cambiamento ${ }^{10}$.

\section{Addomesticamento del veganismo}

La diffusione di stereotipi e pregiudizi sui vegani innalza resistenze contro il veganismo ed è facilitata dalla debolezza del movimento rispetto ai media. Ma non è questa l'unica sfida per il veganismo. Negli ultimi anni si è aggiunta una forma di resistenza meno appariscente ma non per questo meno efficace. Anzi, tanto più efficace in quanto meno visibile. Si tratta dell'ingresso del veganismo nel mercato dei consumi di massa.

Non pochi hanno salutato tale debutto come il segno di una sempre più vicina vittoria del veganismo sull'industria della carne e sull'ideologia del dominio umano sull'animale. In realtà, può essere più correttamente interpretato come l'assorbimento del veganismo nel meccanismo dei consumi e della moda, che ne disinnesca significato e potenziale rivoluzionari per meglio adattarlo ai gusti del mercato di massa e trarne il maggiore profitto possibile ${ }^{11}$. Questo processo di integrazione commerciale del veganismo non è del tutto nuovo. Nel caso del vegetarianismo inglese è stato osservato qualche decennio $\mathrm{fa}^{12}$. È inoltre del tutto coerente con una rappresentazione del veganismo come dieta salutista - ovviamente quando non seguita troppo a lungo - che si è diffusa negli ultimi anni sui mass media e che rappresenta, in fondo, una delle vere ragioni della sua popolarità sui mezzi di comunicazione di massa ${ }^{13}$.

Tutto torna, dunque: se il veganismo è stato accolto e integrato nella cultura, sui media e sul mercato, non lo è stato come scelta etica, ma come tendenza dietetica. Il tutto a spese della marginalizzazione delle sue istanze politiche che, quando non taciute, continuano ad essere oggetto di stigma.

L'insofferenza per la dimensione etico-politica del veganismo non va ascritta unicamente all'antropocentrismo, che pure gioca un ruolo importante, ma anche al carattere individualista, secolarizzato e consumista della cultura occidentale contemporanea. Questa, al di fuori di pochi e generalissimi valori riconosciuti di

\footnotetext{
${ }^{10}$ Cfr. N. Righetti, «L’inchiostro digitale è vegano?», cit., pp. 191-192.

${ }^{11}$ Cfr. Id., «Il Veganismo tra mainstream e controcultura», in «Micro \& Macro Marketing», n. 1, aprile 2018.

12 Cfr. Alan Beardsworth e Eric T. Keil, «Contemporary Vegetarianism in the U.K.: Challenge and Incorporation?», in «Appetite», vol. 20, 1993, pp. 229-234.

${ }^{13}$ Cfr. N. Righetti, «L'inchiostro digitale è vegano?», cit.
} 
importanza collettiva, scaccia nel privato delle opzioni soggettive qualunque ideale di rinnovamento morale della società ${ }^{14}$. L'attuale contesto sociale è in grado di integrare il veganismo soltanto come dietetica che non include un'agenda etica e politica ${ }^{15}$. Quando questa emerge, l'insofferenza si manifesta nelle tradizionali modalità marginalizzanti. Il vegano viene immediatamente ricondotto a un fanatico - termine che non a caso rinvia alla semantica religiosa ${ }^{16}$ - coerentemente a una visione svalutante della vita animale che immediatamente etichetta chi la difende come un individuo che presta un'attenzione esagerata a qualcosa che non ne merita così tanta ${ }^{17}$.

Dato questo contesto si comprende meglio il profilo, affatto casuale, assunto dal veganismo sui media. Da un lato, ospite d'onore nelle rubriche dedicate a cucina, benessere e moda cruelty-free. In questo modo si sono create le opportunità per la promozione di nuovi prodotti. Dall'altro, attaccato sulle pagine di cronaca per le insidie che nasconderebbe la sua alimentazione, quando seguita troppo a lungo e con continuità, inequivocabile segno di un'adesione fanatica e malsana al veganismo. Un'adesione che contrasterebbe con le presunte esigenze "naturali" dell'organismo ${ }^{18}$, ma che contrasta ancor più con quelle del mercato, che apprezza i consumatori che traggono vantaggio dall'intero ventaglio di prodotti loro offerto, senza escluderne intere gamme.

Il messaggio comunicato in questo modo è chiaro: il consumo di prodotti vegan va bene, anzi è persino chic, ma è pericolosa e da condannare l'adesione "ideologica" al veganismo. Ridotto a quindi a dietetica depurativa, a tendenza dell'ultima ora, entrato nei supermercati e nella vita quotidiana in forma politicamente neutralizzata, il veganismo si trova disarmato e addomesticato dai mass media e dal mercato, assoggettato a una ridefinizione del suo significato sempre più indipendente dai suoi autentici obiettivi. Nasce così una forma di veganismo del tutto inoffensiva, un veganismo commerciale in cui la dietetica salutista assume un'importanza fondamentale. La spoliazione politica appare evidente persino sulle confezioni dei

\footnotetext{
${ }^{14}$ Cfr. Id., «Tra sacro e vegano», cit.

${ }^{15}$ Cfr. Jessica B. Greenebaum, «Managing Impressions: "Face-Saving" Strategies of Vegetarians and Vegans», in «Humanity \& Society», vol. 36, n. 4, 2012, pp. 309-325 (in particolare p. 310).

16 Dal latino fanatǐcus «ispirato», der. di fanum «tempio» (Cfr. N. Righetti, «Tra sacro e vegano», Ferrari Editore, Corigliano - Rossano 2018)

${ }^{17}$ In una ricerca di carattere esplorativo si è trovato che il $60 \%$ dei rispondenti non vegani né vegetariani $(\mathrm{N}$ = 222) si trovavano d'accordo con la seguente opinione: "In generale, i vegani sono dei fanatici" (Cfr. N. Righetti, «Il Veganismo tra mainstream e controcultura», cit.)

${ }^{18} \mathrm{Cfr}$. N. Righetti, «L'inchiostro digitale è vegano?», cit.
} 
prodotti vegani in commercio: nel loro classico e rassicurante colore verde, rinviano ai mondi della natura e del benessere, senza nessun esplicito riferimento alla questione animale. Il veganismo mainstream è un veganismo senza derivati di origine animalista.

Così, prima i mass media e poi la comunicazione promozionale, in modi diretti e indiretti, hanno assunto il potere di definire l'identità pubblica del veganismo. Un'identità che quest'ultimo non ha saputo forgiare per mancanza di organizzazione e risorse. Ma oltre all'evidente spoliazione etico-politica del veganismo, la sua commercializzazione potrebbe produrre ulteriori effetti, tutti ancora da indagare. A titolo d'ipotesi: la disponibilità di prodotti vegani nei supermercati potrebbe smorzare la carica critica di alcuni vegani. Nella ricerca di prodotti cruelty-free il vegano sacrifica tempo ed energia per agire coerentemente ai propri valori, così quotidianamente riaffermandoli e leggendo, nell'assenza dei prodotti ricercati, la consapevolezza dell'assenza sociale dei valori che vuole affermare. L'avere a portata di mano i prodotti vegan-friendly potrebbe allora moderare il suo impulso al cambiamento, sopire la sua fame e sete di giustizia. La società, avendo risposto ai bisogni della sua coscienza, potrebbe apparirgli non più così "cattiva" e, forse, già sulla strada del cambiamento, senza bisogno di ulteriori interventi individuali od organizzati. Con la mutazione dei vegani, da rivoluzionari arrabbiati a consumatori appagati, il processo di integrazione del veganismo sarebbe allora completo, e l'ideale del veganismo commerciale trasformato in realtà.

\section{Per concludere}

In questo contributo si è posta l'attenzione su due strategie di resistenza al veganismo. La prima impedisce al veganismo di diffondere il suo messaggio mediante una costellazione di stereotipi chiamata vegefobia. La seconda incorpora il veganismo all'interno del mercato, escludendone le componenti etiche, politiche e critiche, creando un veganismo commerciale ed addomesticato.

Entrambe le strategie si intrecciano e rafforzano reciprocamente come parti di uno stesso processo, che trova le sue condizioni di possibilità nella cultura antropocentrica, individualista, secolarizzata e consumista, ma anche nelle caratteristiche del veganismo stesso.

Difatti, la costitutiva mancanza di organizzazione del veganismo ne determina l'impotenza nei confronti dei mass media, rispetto ai quali si trova nella condizione di 
oggetto più che di soggetto del discorso. Nonostante ci siano molti altri canali di comunicazione, come quelli digitali, i mass media tradizionali hanno ancora un ruolo di primaria importanza e negli ultimi anni sono stati il veicolo di trasmissione di notizie che hanno gettato discredito sul veganismo. Quest'ultimo non ha potuto replicare in nessun modo, in quanto privo, per sua natura, di rappresentanti accreditati. Le analisi svolte sulla stampa hanno mostrato che lo stesso non accade per i movimenti animalisti più importanti, caratterizzati da una struttura organizzativa consolidata in grado di veicolare la propria voce sui media.

Rispetto ai rapporti con i media, il caratteristico individualismo del movimento vegano rappresenta dunque un punto di debolezza, in quanto lo rende incapace di svolgere funzioni di controllo, per quanto parziali, sulla propria immagine pubblica, che può così essere manipolata dal sistema mediatico producendo conseguenze deleterie.

Anche l'integrazione nel mercato è coerente con la centralità dei consumi nella strategia vegana. In modo paradossale rispetto agli obiettivi del veganismo, ma anche del tutto logico, il mercato ha creato prodotti adatti al consumo cruelty-free, senza modificare in nulla il proprio rapporto con gli animali, ma semplicemente affiancando linee di prodotti vegan-friendly a quelle tradizionali.

Non solo, quindi, il veganismo non è riuscito a trasformare il sistema, ma è stato, per così dire, esso stesso trasformato e manipolato dal sistema. Questo porta a chiedersi se il tipo di attivismo proposto dal veganismo sia sufficiente allo scopo che si prefigge o non debba essere ripensato.

Negli ultimi anni, certo, non sono mancati nemmeno dei buoni risultati. La popolarità del veganismo ha contribuito a far conoscere alcuni temi animalisti. In modo spesso disfunzionale, è vero, ma nondimeno questa popolarità rappresenta un capitale da valorizzare. Una strategia condivisa, tuttavia, sembra la condizione minima perché questo possa accadere.

\section{Bibliografia}

Beardsworth, A., Kei, E.T. (1993). Contemporary Vegetarianism in the U.K.: Challenge and Incorporation?, Appetite, vol. 20, pp. 229-234.

Cole, M., Morgan, K. (2011). Vegaphobia: Derogatory Discourses of Veganism and the Reproduction of Speciesism in UK National Newspapers, British Journal of Sociology, n. 62, vol. 1, pp. 134-153 (trad. it. in 
http://it.vegephobia.info/index.php?post/2012/05/21/Vegafobia-di-M.Cole-eK.-Morgan).

Eurispes (2017), Rapporto Italia 2017.

Eurispes (2018), Rapporto Italia 2018.

Gamson, W.A., Wolfsfeld, G. (1993). Movements and Media as Interacting Systems, in The Annals of the American Academy of Political and Social Science, n. 528, pp. 114-125.

Greenebaum, J.B. (2012). Managing Impressions: "Face-Saving" Strategies of Vegetarians and Vegans, Humanity \& Society, vol. 36, n. 4, 2012, pp. 309-325.

Haenfler, R., Johnson, B., Jones, E. (2012). Lifestyle Movements: Exploring the Intersection of Lifestyle and Social Movements, Social Movement Studies, vol. 11, n. 1, pp. 1-20.

Righetti, N. (2016). L'inchiostro digitale è vegano? La rappresentazione del veganismo sulla stampa, Cambio. Rivista Sulle Trasformazioni Sociali, n. 11, pp. 181-194.

Righetti, N. (2018). Tra sacro e vegano, Ferrari Editore, Corigliano - Rossano.

Righetti, N. (2018). Il Veganismo tra mainstream e controcultura, Micro \& Macro Marketing, n. 1, aprile.

The Vegan Society (2014). Ripened by human determination. 70 years of The Vegan Society,https://www.vegansociety.com/sites/default/files/uploads/Ripened $\% 20$ by $\% 20$ human $\% 20$ determination.pdf.

Yinger, J.M. (1960). Contraculture and Subculture, American Sociological Review, vol. 25, n. 5, 1960, pp. 625-635. 